\title{
Ion-Pair Evaporation from Ionic Liquid Clusters
}

\author{
Christopher J. Hogan Jr. ${ }^{\mathrm{a}, \mathrm{b}, *}$ and Juan Fernandez de la Mora ${ }^{\mathrm{a}}$ \\ ${ }^{a}$ Department of Mechanical Engineering, Yale University, New Haven, Connecticut, USA \\ b SEADM, Boecillo, Spain
}

A differential mobility analyzer (DMA) is used in atmospheric pressure $\mathrm{N}_{2}$ to select a narrow range of electrical mobilities from a complex mix of cluster ions of composition $(C A)_{n}\left(C^{+}\right)_{\mathrm{z}}$. The clusters are introduced into the $\mathrm{N}_{2}$ gas by electrospraying concentrated $(\sim 20 \mathrm{mM})$ acetonitrile solutions of ionic liquids (molten salts) of composition $\mathrm{CA}\left(\mathrm{C}^{+}=\right.$cation, $\mathrm{A}^{-}=$ anion). Mass analysis of these mobility-selected ions reveals the occurrence of individual neutral ion-pair evaporation events from the smallest singly charged clusters: $(\mathrm{CA})_{n} \mathrm{C}^{+} \rightarrow(\mathrm{CA})_{n-1} \mathrm{C}^{+}+\mathrm{CA}$. Although bulk ionic liquids are effectively involatile at room temperature, up to six sequential evaporation events are observed. Because this requires far more internal energy than available in the original clusters, substantial heating $(\sim 10 \mathrm{eV})$ must take place in the ion guides leading to the mass analyzer. The observed increase in IL evaporation rate with decreasing size is drastic, in qualitative agreement with the exponential vapor pressure dependence predicted by Kelvin's formula. A single evaporation event is barely detectable at $n=13$, while two or more are prominent for $n \leq 9$. Magic number clusters $(\mathrm{CA})_{4} \mathrm{C}^{+}$with singularly low volatilities are found in three of the four ionic liquids studied. Like their recently reported liquid phase prenucleation cluster analogs, these magic number clusters could play a key role as gas-phase nucleation seeds. All the singularly involatile clusters seen are cations, which may help understand commonly observed sign effects in ion-induced nucleation. No other charge-sign asymmetry is seen on cluster evaporation. (J Am Soc Mass Spectrom 2010, 21, 1382-1386) (c 2010 American Society for Mass Spectrometry

$\mathrm{W}$ e have recently used a differential mobility analyzer (DMA) [1-3] with a mass spectrometer (MS) to examine ionic liquid clusters of composition $(\mathrm{CA})_{n}\left(\mathrm{C}^{+}\right)_{z}$ [4]. The DMA is a narrow band mobility filter which can be used to select parent ions and monitor their transitions with a second analyzer. Although our work with ionic liquid clusters [4] did not actively excite ion fragmentation, the mass spectra of DMA-selected ions showed a diversity of product ions along with the parent ion, clearly revealing individual neutral ion-pair evaporation events of the form $(\mathrm{CA})_{n} \mathrm{C}^{+} \rightarrow(\mathrm{CA})_{n-1} \mathrm{C}^{+}+\mathrm{CA}$. Metastable evaporation events from ionic clusters have been previously observed for hot ions formed by highenergy processes, in particular sputtering from CsI surfaces $[5,6]$. However, these are the first direct observations of single molecule evaporation events with ionic liquids, and therefore warrant the present more detailed account.

Address reprint requests to Dr. J. Fernandez de la Mora, Department of Mechanical Engineering, Yale University, New Haven, CT 06520-8286, USA. E-mail: juan.delamora@yale.edu

* Current address: Department of Mechanical Engineering, University of Minnesota, Minneapolis, MN 55455, USA.

\section{Experimental}

\section{Ionic Liquid Cluster Production}

The ionic liquids 1-ethyl-3-methyl-imidazolium ${ }^{+}$(EMI)tris(trifluoromethylsulfonyl) methide ${ }^{-}$(EMI-Methide), EMI-bis(trifluoromethylsulfonyl)imide (EMI-Im), and EMI-bis(perfluoroethylsulfonyl)imide (EMI-Beti), were purchased from Covalent Associates (Washburn, MA, USA). EMI-BF $F_{4}$ was from Fluka. Each was mixed with acetonitrile at IL concentrations of $20 \mathrm{mM}$, and electrosprayed (in both polarities) using a $50 \mu \mathrm{m}$ i.d. silica capillary as described previously [4].

\section{Differential Mobility Analysis-Mass Spectrometry}

DMA-MS operation is described elsewhere [3, 4], and a schematic of the DMA-MS is shown in the Supplementary Information, which can be found in the electronic version of this article. Briefly, IL clusters were drawn electrostatically into a parallel plate DMA (model P4; SEADM, Boecillo, Spain). The DMA was operated in recirculating mode with dry $\mathrm{N}_{2}$ as the sheath gas. Dry $\mathrm{N}_{2}$ was injected into the closed gas circuit to replace the gas sampled into the mass spectrometer $(0.4 \mathrm{~L} / \mathrm{min})$ and provide a small counterflow gas $(\sim 0.2 \mathrm{~L} / \mathrm{min})$ that exited the DMA through its sample inlet slit into the source region. This ensured that no vapors or $\mathrm{CO}_{2}$ (used 
to maintain a stable electrospray) entered into the DMA, which operated with dry $\mathrm{N}_{2}$. DMA voltages were scanned in the 2000-5500 V range in increments of $5 \mathrm{~V}$ to collect mobility spectra. The DMA voltage at which an ion is transmitted is inversely proportional to its electrical mobility $Z$, with a proportionality constant fixed for a given experiment. The value of this constant was determined by means of a calibration standard yielding absolute mobilities. The DMA was coupled to a QSTAR XL mass spectrometer (Applied Biosystems, Framingham, MA, USA) as described in an earlier report [4]. Declustering potentials in the mass spectrometer inlet were set close to their minimum values of $1 \mathrm{~V}$ $(\mathrm{DP}=\mathrm{FP}=\mathrm{DP} 2=1 \mathrm{~V} ; \mathrm{IRD}=6 \mathrm{~V} ; \mathrm{IRW}=5 \mathrm{~V})$.

\section{Results and Discussion}

\section{Individual Ion-Pair Evaporation of Clusters}

Figure 1 shows cluster abundance versus inverse electrical mobility, $1 / Z$ (horizontal variable), and $n / z$ (number of neutral ion pairs over net charge on the vertical axis) for positively charged cluster ions of EMIMethide. The $n / z$ variable is convenient for cluster identification and is calculated as:

$$
n / z=\frac{m / z-m_{C}}{m_{C}+m_{A}},
$$

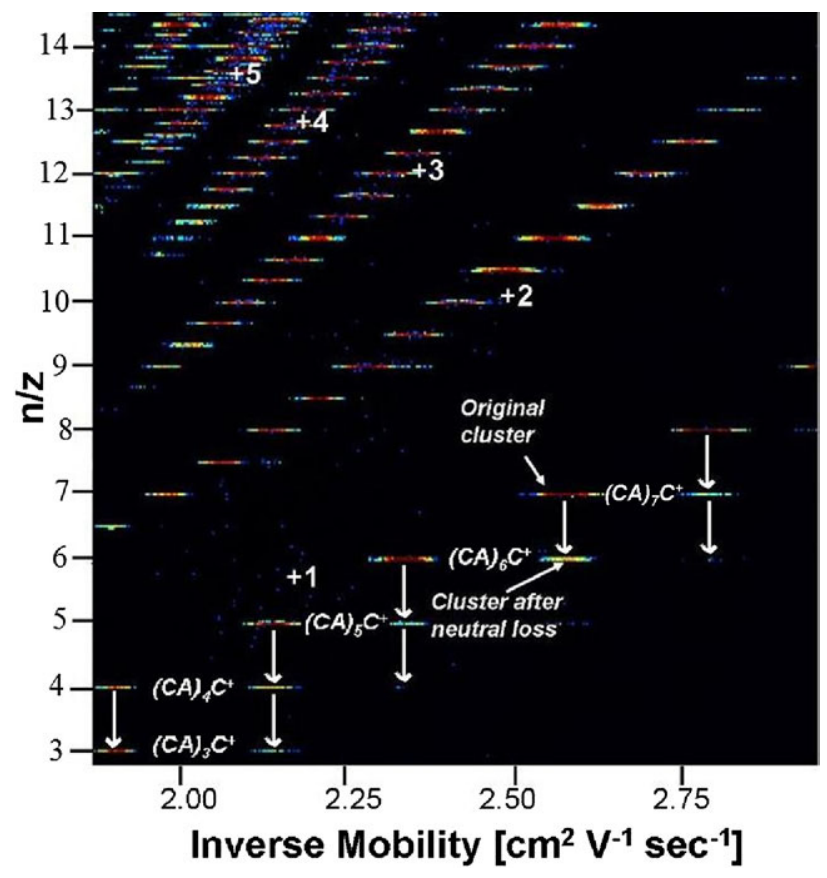

Figure 1. A differential mobility analyzer-mass spectrometer (DMA-MS) spectrum of positively charged EMI-methide clusters. The charge state of each group of clusters is labeled on the plot, as are the cluster formulae, i.e., $\left(\mathrm{CA}_{\mathrm{x}}\right) \mathrm{C}^{+}(\mathrm{C}=$ cation, $\mathrm{A}=$ anion $)$, for selected singly charged clusters. The arrows mark neutral evaporation events taking place between the mobility and the mass measurement. where $m_{C}$ and $m_{A}$ are the masses of the cation and the anion, respectively (note $n / z=\left(m / z-m_{A}\right) /\left(m_{C}+m_{A}\right)$, for negatively charged clusters). The measured pair $(Z, n / z)$ yields $n$ and $z$. Multiply charged clusters $(z>1)$ are abundant in Figure 1 because declustering potentials, which promote the dissociation of multiply charged clusters, were not employed during measurements [4]. Each peak (colored horizontal segment) is associated to a cluster $(\mathrm{CA})_{n} \mathrm{C}^{+}{ }_{z}$, with the prominent inclined bands in the figure grouping clusters with the same $z$ (see figure labels) and varying $n$. All but one of these bands includes a single peak in the mass spectrum for each mobility. The exception is the $z=1$ band, in which multiple vertically displaced mass peaks with structure $(\mathrm{CA})_{n} \mathrm{C}^{+},(\mathrm{CA})_{n-1} \mathrm{C}^{+},(\mathrm{CA})_{n-2} \mathrm{C}^{+}, \ldots$, appear at exactly the same mobility, like rungs in a ladder. Similar mysterious columnar structures have been previously observed with cluster ions from ammonium salts [7]. Using the mass-mobility relation for spherical nanoparticles [8], we see that only the top rung in each of these ladders has the correct mass for the given mobility. We thus conclude that the anomalous lower rungs are decay products from a single stable parent ion selected by the DMA. This parent ion is heated somewhere between the two analyzers, leading to one or several neutral pair evaporation events before mass analysis:

$$
(C A)_{n} C^{+} \rightarrow(C A)_{n-1} C^{+}+C A
$$

The evidence for heating downstream the DMA is multiple. The parent ion is completely stable at room temperature in the DMA. Otherwise the mobility peaks would not be sharp. The drift velocity for these ions is 10-20 m/s, implying much larger residence times (700 $\mu$ s for a $1 \mathrm{~cm}$ gap between electrodes) in the DMA than in the MS. Furthermore, the typical heat of vaporization in ionic liquids $(1.5 \mathrm{eV})$ [9] is larger than the internal energy available in a cluster with $n \sim 9$. Multiple evaporation events therefore require external heat not available in a cold gas jet. At DV $=1$, the only heating source present is the RF field. Because there are no ion-neutral collisions in the analyzing quadrupole, this heating must take place in the ion guide Q0. Independent control of the voltages in Q0 is possible but experimentally nontrivial and was not pursued, so the temperature at which the process is taking place is unknown.

Although bulk evaporation of several ionic liquids has been observed in distillation experiments [10, 11], the ladders of Figure 1 provide the most direct available evidence for the expected mechanism (2) for IL evaporation. Prior indirect evidence has been obtained via electron bombardment of neutral volatiles released from heated bulk ILs [9]. Although the expected ionization process is $\mathrm{CA}+\mathrm{e}^{-} \rightarrow 2 \mathrm{e}^{-}+(\mathrm{CA})^{+}$, the dominant ion found in these studies was $\mathrm{C}^{+}$, with no trace of $(\mathrm{CA})^{+}$. 
This is attributed to instability of $(\mathrm{AC})^{+}$, which dissociates as [9]:

$$
\mathrm{CA}+e^{-} \rightarrow(\mathrm{CA})^{+}+2 e^{-} \rightarrow \mathrm{C}^{+}+A+2 e^{-}
$$

Instability of the positively charged ion pair following photoionization [12] and field ionization [13] of the neutral vapor has also been reported. Hence, with the exception of the detection of $(\mathrm{CA})^{+}$at $\sim 1 \%$ of the abundance of $\mathrm{C}^{+}$for one of the four ILs studied by Gross [13] [EMI-C(CN $)_{3}$ ], the only observable ion was not directly connected to ion-pair evaporation in previous studies. In electrospray DMA-MS, however, only one reaction (eq 2) takes place, and all the ions involved in each of its occurrences are observed.

\section{Singularly Involatile Clusters}

Figure 2 shows DMA-MS spectra for EMI-BF 4 , EMI-Im, and EMI-Beti, respectively, all in positive polarity, and all exhibiting evaporation of ion-pairs. Similar results are obtained with negatively charged clusters. The number of discrete $(\mathrm{CA})_{n} \mathrm{C}^{+} \rightarrow(\mathrm{CA})_{n-1} \mathrm{C}^{+}+\mathrm{CA}$ events that occur between the DMA-MS increases drastically with decreasing cluster size. Ion-pair evaporation is barely detectable for EMI-Methide clusters with $n=13$, but multiple evaporation events occur for clusters with $n \leq 9$. Most striking in Figure 2a-c is the singularly low volatility of all positively charged $n=4$ clusters. Conversely, abnormally high volatility is seen for doubly charged EMI-Methide clusters with $n=16$ and 19, which decay by loss of a single ion pair. No unusually volatile or involatile clusters were apparent in negative mode. Unlike cluster abundance measured by MS alone [14], here the measured abundance of each cluster is directly related to the cluster's evaporation rate relative to its neighbors.

\section{Nucleation Implications}

The existence of stable clusters substantially larger than the monomer vapor molecule provides an energy saving bridge in nucleation problems. This point has been recently highlighted for liquid phase nucleation of $\mathrm{CaCO}_{3}$ via prenucleation clusters apparently with $n \sim$ 70 [15]. Such stable clusters have been more clearly observed with $n=12-13$ in concentrated aqueous solutions of sucrose [16]. Evidently, the common characteristic between such stable liquid phase prenucleation clusters and gas-phase magic number clusters (which are not stable at the vanishing monomer concentration investigated here) is their singular stability against dissolution or evaporation, enabling them to exist as permanent entities at monomer concentrations at which larger or smaller clusters (except the monomer) are unstable. High magic number cluster abundances have been recently linked also with homogeneous nucleation [17].

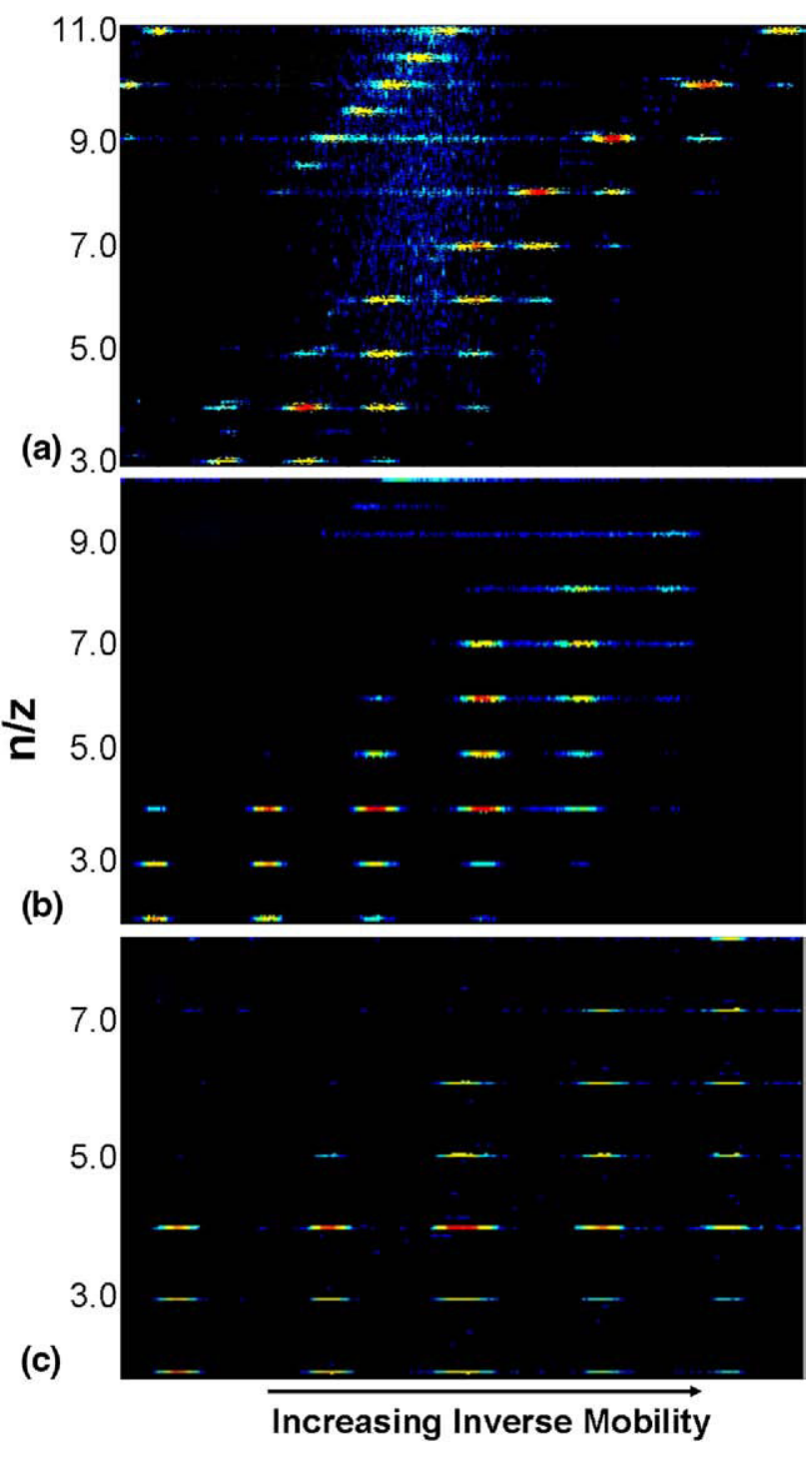

Figure 2. DMA-MS spectrum for +1 charged clusters of the ionic

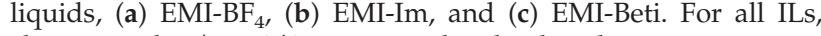
clusters with $n / z=4 / 1$ are anomalously abundant.

\section{Neutral Evaporation Kinetics}

Measured cluster signal intensities were used to obtain normalized neutral pair evaporation rate constants, $k_{n}$ [equation (4a)] as functions of the number of ion-pairs per cluster. The inversion routine used to determine the $k_{n}$ is described in the Supplementary Information. The residence time and the cluster temperature in the RF heating zone are unknown but were provisionally taken to be comparable for all clusters; thus, all inferred $k_{n}$ values were normalized such that $k_{11}$ for EMI-Beti clusters was 1.00. Figure 3 shows the inferred $k_{n}$ values for all ILs examined as a function of $n$, the number of ion-pairs in the evaporating cluster. Ion-induced nucleation is dependent on ion polarity [18]; thus, it is important to establish if this peculiarity stems from the condensation or the evaporation rate. This comparison is limited to only two of the ILs (open symbols, positively charged clusters; closed sym- 


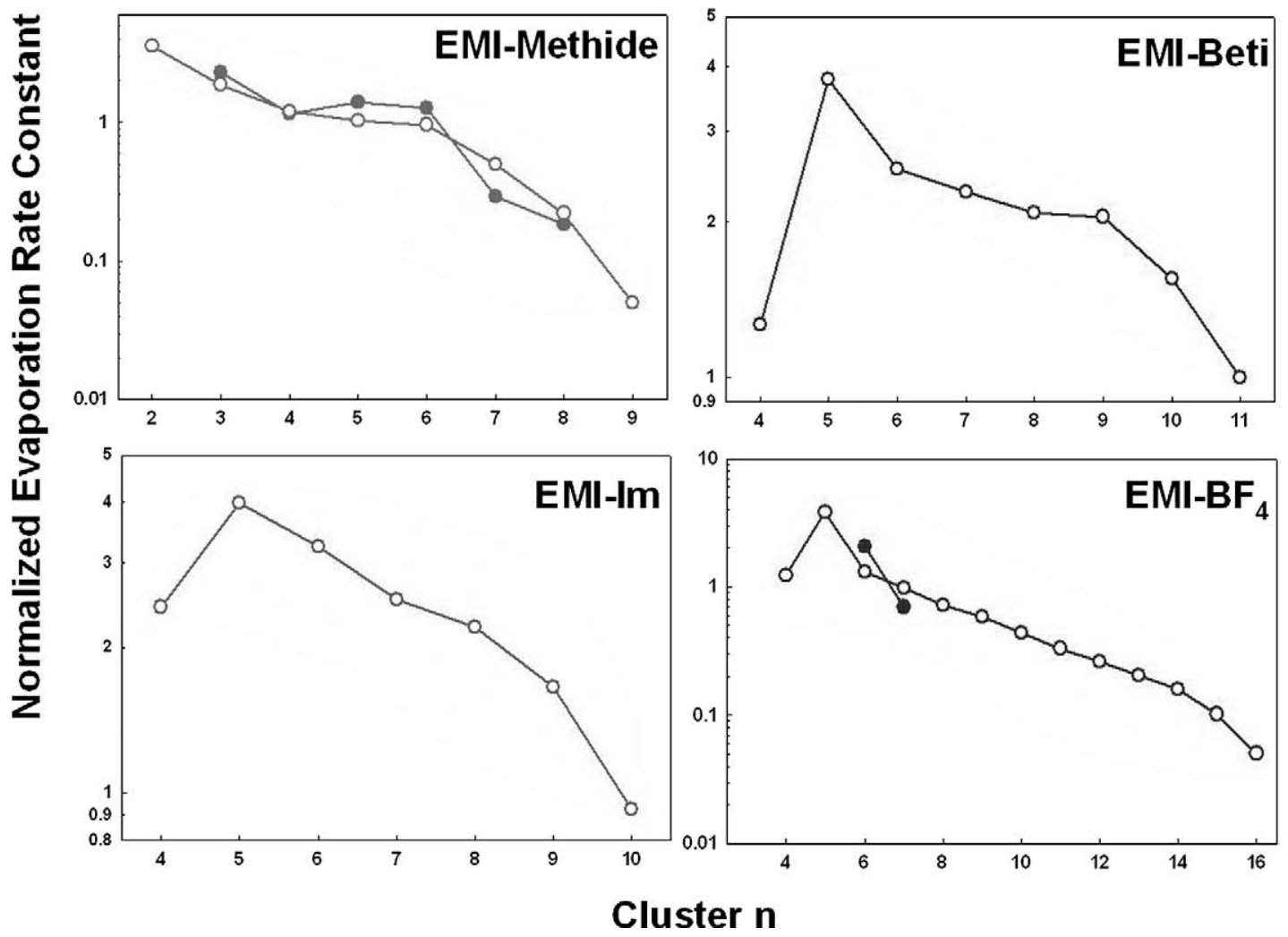

Figure 3. Normalized evaporation rate constants as functions of the number of ion-pairs in each IL. Open symbols $=$ positively charged clusters. Closed symbols $=$ negatively charged clusters.

bols, negative charged clusters in Figure 3) due to the poorer quality of negative spectra [4]. Within these limitations, Figure 3 shows no obvious volatility sign preference. However, all magic number clusters found are cations, and this sign asymmetry could have a drastic effect on ion induced nucleation.

A comparison between inferred evaporation rates and the evaporation rates expected based on the Kelvin equation [19] is given in the Supplementary Information. Briefly, inferred rates are in qualitative agreement with these predictions, in that the evaporation rate increases drastically with decreasing cluster size. However, more detailed studies of cluster dynamics in the inlet of a mass spectrometer, with accurate modeling of $\mathrm{rf}$ heating, need to be performed for rigorous comparison between the Kelvin equation and our measurements.

\section{Conclusions}

The DMA provides a useful tool to isolate mobilityselected ions as produced by an electrospray source. With it, we detect previously unobserved transitions, probably taking place in the inlet region of many other mass spectrometers with atmospheric pressure sources.

RF Heating in the ion guide region of the mass spectrometer used is substantial. It imparts $\sim 10 \mathrm{eV}$ to small nanodrops, and leads to single and multiple neutral evaporation events even for highly involatile ionic materials, captured for the first time here for ionic liquids.

We observe singularly abundant magic number clusters of structure $(\mathrm{CA})_{4} \mathrm{C}^{+}$for 3 of the four ILs investigated. No negatively charged magic number cluster analog was found.

These magic number clusters have anomalously small volatilities, may play a role in nucleation phenomena, and provide a new potential mechanism to explain observed charge asymmetry in ion induced nucleation.

\section{Acknowledgments}

The authors thank Mr. Juan Rus and Mr. David Moro for setting up and maintaining the DMA-MS system, and Mr. Alejandro Casado for developing the software used in data collection and processing. All experiments reported were performed at SEADM's laboratory (Boecillo, Spain), and were funded by SEADM, with additional contributions to data interpretation provided by AFOSR grant FA9550-06-1-0104.

\section{Appendix A Supplementary Material}

Supplementary material associated with this article may be found in the online version at doi:10.1016/ j.jasms.2010.03.044. 


\section{References}

1. Fernandez de la Mora, J.; de Juan, L.; Eichler, T.; Rosell, J. Differential Mobility Analysis of Molecular Ions and Nanometer Particles. Trends Anal. Chem. 1998, 17(6), 328-339.

2. Knutson, E. O.; Whitby, K. T. Aerosol Classification. by Electric Mobility: Apparatus, Theory, and Applications. J. Aerosol Sci. 1975, 6, 443-451.

3. Rus, J., Moro, D., Sillero, J. A., Royuela, J., Casado, A., Estevez-Molinero, F. Fernandez de la Mora, J. IMS-MS Studies Based on Coupling a Differential Mobility Analyzer (DMA) to Commercial API-MS Systems. Int. J. Mass Spectrom. 2010, unpublished, (submitted).

4. Hogan, C. J.; Fernandez de la Mora, J. Tandem Ion Mobility-Mass Spectrometry (IMS-MS) Study of Ion Evaporation from Ionic LiquidAcetonitrile Nanodrops. Phys. Chem., Chem. Phys. 2009, 11, 80798090 .

5. Ens, W.; Beavis, R.; Standing, K. G. Time-of-Flight Measurements of Cesium-Iodide Cluster Ions. Phys. Rev Lett. 1983, 50(1), 27-30.

6. Drewello, T.; Herzschuh, J.; Stach, J. Direct Fission Versus Sequential Evaporation Mechanism of Sputtered Caesium Iodide Cluster Ions. Z Phys. D 1993, 28(4), 339-343.

7. Trimpin, S.; Clemmer, D. E. Ion Mobility Spectrometry/Mass Spectrometry Snapshots for Assessing the Molecular Compositions of Complex Polymeric Systems. Anal. Chem. 2008, 80(23), 9073-9083.

8. Ku, B. K.; Fernandez de la Mora, J. Relation Between Electrical Mobility, Mass, and Size for Nanodrops 1-6.5 nm in Diameter in Air. Aerosol Sci. Technol. 2009, 43(3), 241-249.
9. Armstrong, J. P.; Hurst, C.; Jones, R. G.; Licence, P.; Lovelock, K. R. J. Satterley, C. J.; Villar-Garcia, I. J. Vaporization of Ionic Liquids. Phys. Chem., Chem. Phys. 2007, 9, 982-990.

10. Earle, M. J.; Esperanca, J. M. S. S.; Gilea, M. A.; Lopes, J. N. C.; Rebelo, L. P. N.; Magee, J. W.; Seddon, K. R.; Widegren, J. A. The Distillation and Volatility of Ionic Liquids. Nature 2006, 439(7078), 831-834.

11. Wasserscheid, P. Chemistry-Volatile Times for Ionic Liquids. Nature 2006, 439(7078), 797-797.

12. Strasser, D.; Goulay, F.; Kelkar, M. S.; Maginn, E. J.; Leone, S. R Photoelectron Spectrum of Isolated Ion-Pairs in Ionic Liquid Vapor. J. Phys. Chem. A 2007, 111(17), 3191-3195.

13. Gross, J. H. Molecular Ions of Ionic Liquids in the Gas Phase. J. Am. Soc. Mass Spectrom. 2008, 19(9), 1347-1352.

14. Sakurai, M.; Watanabe, K.; Sumiyama, K.; Suzuki, K. Magic Numbers in Transition Metal ( $\mathrm{Fe}, \mathrm{Ti}, \mathrm{Zr}, \mathrm{Nb}$, and $\mathrm{Ta}$ ) Clusters Observed by Timeof-Flight Mass Spectrometry. J. Chem. Phys. 1999, 111(1), 235-238.

15. Gebauer, D.; Volkel, A.; Colfen, H. Stable Prenucleation. Calcium Carbonate Clusters. Science 2008, 322(5909), 1819-1822.

16. Kaufman, S. L.; Dorman, F. D. Sucrose Clusters Exhibiting a Magic Number in Dilute Aqueous Solutions. Langmuir 2008, 24(18), 9979-9982.

17. Girshick, S. L.; Agarwal, P.; Truhlar, D. G. Homogenous Nucleation with Magic Numbers: Aluminum. J. Chem. Phys. 2009, 131, 134305/1-11.

18. Seto, T.; Okuyama, K.; deJuan, L.; Fernandez de la Mora, J. Condensation of Supersaturated Vapors on Monovalent and Divalent Ions of Varying Size. J. Chem. Phys. 1997, 107(5), 1576-1585.

19. Friedlander, S. K. Smoke, Dust, and Haze; Oxford University Press: New York, 2000; p. 257, Eq. 9.17. 